\title{
AUGMENTED REALITY BASED DOPPLER LIDAR DATA VISUALIZATION: Promises AND CHALLENGES \\ Cherukuru, N. W., ${ }^{1 *}$ and Calhoun, R. ${ }^{1}$

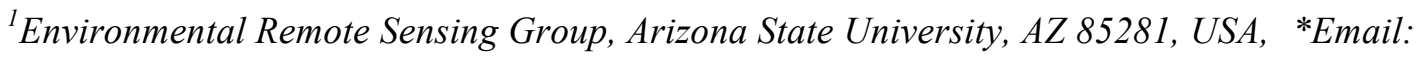 ncheruku@asu.edu
}

\begin{abstract}
Augmented reality (AR) is a technology in which the enables the user to view virtual content as if it existed in real world. We are exploring the possibility of using this technology to view radial velocities or processed wind vectors from a Doppler wind lidar, thus giving the user an ability to see the wind in a literal sense. This approach could find possible applications in aviation safety, atmospheric data visualization as well as in weather education and public outreach.
\end{abstract}

As a proof of concept, we used the lidar data from a recent field campaign and developed a smartphone application to view the lidar scan in augmented reality. In this paper, we give a brief methodology of this feasibility study, present the challenges and promises of using AR technology in conjunction with Doppler wind lidars.

\section{INTRODUCTION}

Augmented reality (AR) is a technology in which 3 -D virtual content is integrated with visual content and presented to the user. The virtual content could be anything that the user cannot directly perceive with his own senses [1]. Based on the technology used to present the virtual content, AR technologies have been classified into two categories. In the first, the virtual content is presented as an overlay on the live camera feed, and displayed on a screen. The second category uses see through Head mounted displays (HMD) in which the virtual content is placed in front of the user's eyes with the help of optical combiners [1]. In the current study, we explore the potential applications of AR technology in conjunction with Doppler lidar which could enable the user to view the surrounding wind fields in an intuitive manner.

There have been a number of studies discussing the capabilities of Doppler lidars in detection of wind shear events, wake vortices, microbursts and other hazardous situations to aircraft and rotorcrafts to name a few [2][3][4]. Previous flight simulation studies on helicopter pilots have shown that visualization of airflow improved the helicopter pilot's landing performance under stressful operational conditions [5].

To test the feasibility of AR technology with Doppler lidars we first implemented this method with a smartphone. Section 2 gives a brief description of the methodology. The results are discussed in section 3 along with the challenges identified in the process. Finally, we conclude with the description of the advantages of using this method on alternate platforms.

\section{METHODOLOGY}

There are four important components in the AR system that we implemented for viewing Lidar data in this test study: a) Location, attitude (roll, pitch and yaw) and heading sensors, b) a processor c) camera and d) display.

Almost all smartphones come equipped with MEMS gyroscope (Micro-Electromechanical systems) to obtain the attitude data, solid-state compass for heading information and a GPS receiver.

First, the location and attitude sensors determine the GPS location as well as the orientation of the device being used to view the lidar data. The processor then acquires the wind data from the lidar and renders the scan as a Layer object. In future we could use standard graphics libraries like OpenGL to construct the lidar scan data for a more efficient implementation. The image of the lidar scan is then scaled and proper perspective transform is applied based on the orientation of the viewing device. Next, the image of the scan is translated based on the heading information obtained from the compass. The image transformation parameters depend on the focal length of the camera lens, field of view of the camera as well as the resolution of the final 


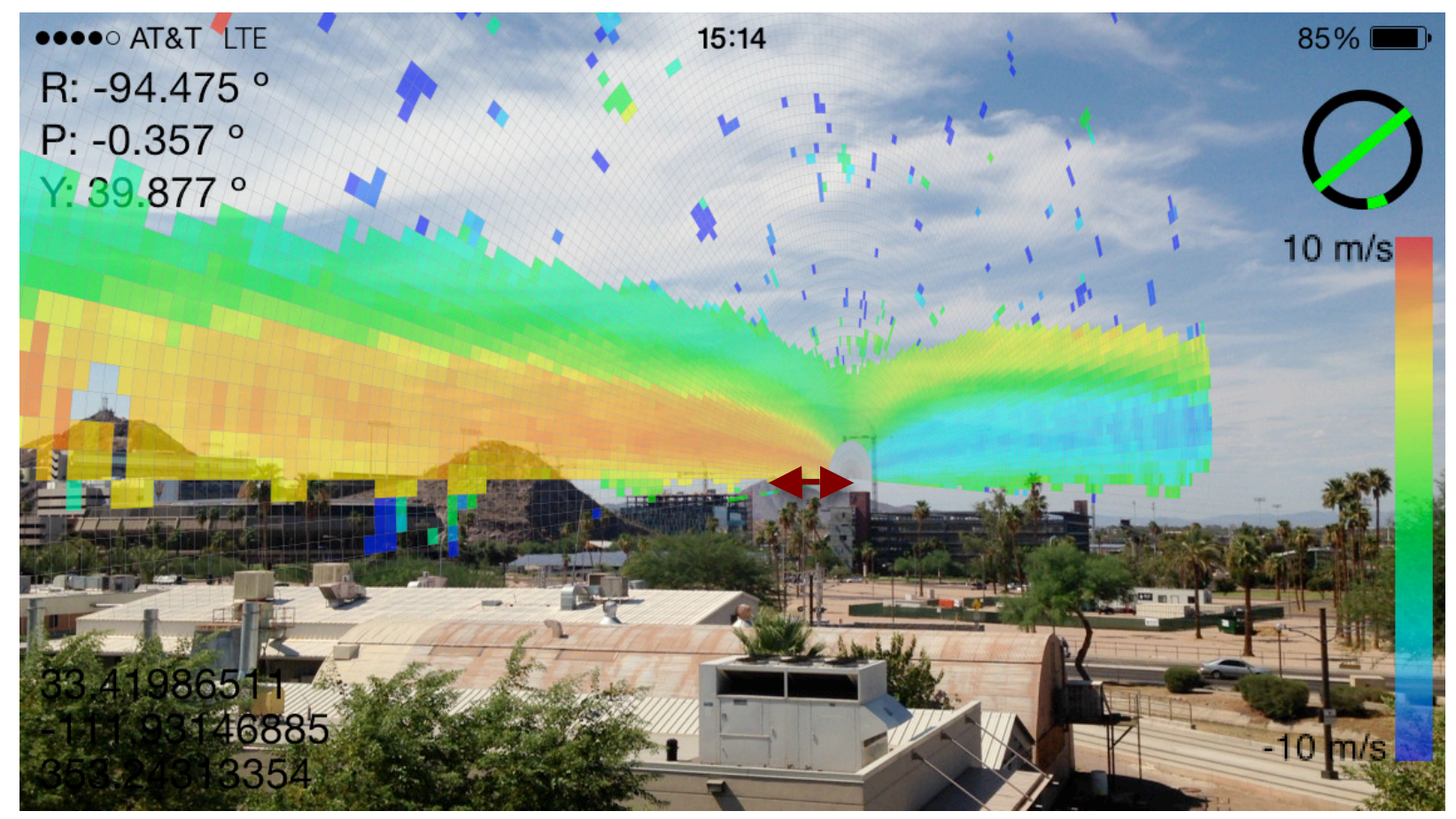

Figure-1. Screenshot of the AR application. The red double headed arrow at the base of the lidar was added to the screenshot after it was captured to show the effect of sensor drift.

display. At this step, the rendered and transformed wind data from the lidar is displayed as an overlay on the live camera feed on the device's screen. The virtual image of the lidar scan is transformed continuously based on the sensor data giving the impression that the scan is fixed at a location in space. This whole system was implemented in Objective C.

The wind data was obtained from a $1.6 \mu \mathrm{m}$ LMCT windtracer lidar. The lidar was virtually placed on a nearby hill that was about $11 \mathrm{~km}$ away from the observation point. The difference in elevations between the location of the virtual lidar and the observation point was $390 \mathrm{~m}$.

\section{RESULTS}

A screen shot of the smartphone was taken while using this application (see figure 1). The RHI (range-height indicator) scan shows the radial velocity at each range gate. ' $R$ ', ' $P$ ', ' $Y$ ' correspond to the roll, pitch and yaw of the device while the screenshot was captured. The colors represent the radial velocity in $\mathrm{m} / \mathrm{s}$. The black circle with a green line and a green dot shows the orientation of the viewer with respect to the lidar scan, i.e., currently, the viewer is looking at the scan from the south and the RHI scan is oriented, NE to SW.

\subsection{Challenges:}

The major challenge was with the accuracy of the sensors used. Although a smartphone can use different methods to obtain the GPS location that may affect the accuracy of the GPS significantly, our observed accuracy seem to conform to the specified $\pm 10 \mathrm{~m}$ range. However, the accuracy can be improved with a commercially available external GPS receiver. The second challenge is with the drift in MEMS gyroscope. All inertial sensors have the issue with drift causing the lidar scan to drift while using the application. Figure 1 also shows the effects of drift and location error of the GPS (red double headed arrow). There have been a few studies to reduce this and correct for the drift programmatically, which could be used in our application [6]. The solid-state compass was sensitive to the proximity of metallic surfaces. While not a major issue, it required calibration from time to time. Although latency measurement between the completion of scan and rendering of the image was not possible with the current setup, we observed that the response of the image to user movements was almost instantaneous. Thus we 
expect the latency to come from the data transfer between the devices, which can be reduced significantly if the visualization is done on the same computer that is analyzing the lidar data.

\section{Promise on alternate platforms:}

\subsection{Video based approach:}

Smartphone will be the most accessible devices for all users but the issue with latency discussed in the challenges section needs to be studied with lidar data collected in real time with data transfer between the lidar and the smartphone

Oculus rift with stereo camera rig could be a feasible option. The main advantage of using oculus rift setup is that it provides an immersive environment and it can also be directly connected with the computer acquiring the data from the lidar. This may help in resolving the latency issue.

\subsection{Optical Technology:}

A well-known device in this category is Google Glass. Although Google is by far the most portable AR glasses, their biggest disadvantage for our lidar application is lack of full screen capabilities. This shortcoming can be met with the recently announced Microsoft HoloLens, a see through head mounted display by Microsoft.

With regard to military applications of the AR system, this technology can be easily integrated with the new head mounted displays like JEDEYETM (from Elbit Systems Ltd.) that currently use the AR technology to help pilots see through brownouts, fog and other low vision conditions [7].

\section{ACKNOWLEDGEMENTS}

The authors would like to thank ASU Lightworks for their support.

\section{REFERENCES}

[1] R. Azuma, August 1995: A survey of augmented reality, Computer Graphics, SIG-GRAPH Proceedings, pp. 1-8.

[2] C. M. Shun and P. W. Chan, 2008: Applications of an Infrared Doppler Lidar in Detection of Wind Shear. J. Atmos. Oceanic Technol., 25, 637-655.
[3] Menzies, Robert T., and R. Michael Hardesty, 1989: Coherent Doppler lidar for measurements of wind fields, Proceedings of the IEEE 77.3: 449462.

[4] Köpp, F., Rahm, S., \& Smalikho, I., 2004: Characterization of Aircraft Wake Vortices by $2-\mu$ $\mathrm{m}$ Pulsed Doppler Lidar. Journal of Atmospheric and Oceanic Technology, 21(2), 194-206.

[5] Aragon, C. R., \& Long, K. R., 2005: Airflow hazard visualization for helicopter pilots: Flight simulation study results. Annual forum proceedings - American Helicopter Society (Vol. 61, No.1, p.1). AMERICAN HELICOPTER SOCIETY, INC.

[6] Hol, J. D., 2011: Sensor fusion and calibration of inertial sensors, vision, ultra-wideband and GPS. Linköping studies in science and technology. dissertations, (1368).

[7] H.-U. Doehler ; Sven Schmerwitz and Thomas Lueken, 2014: Visual-conformal display format for helicopter guidance ", Proc. SPIE 9087, Degraded Visual Environments: Enhanced, Synthetic, and External Vision Solutions. 